\title{
Advanced Gated X-Ray Imagers for Experiments at the National Ignition Facility
}

S. Glenn, P. Bell, L. Benedetti, D. Bradley, J. Celeste, R. Heeter, C. Hagmann, J. Holder, N. Izumi, J. Kilkenny, J. Kimbrough, G. Kyrala, N. Simanovskaia, R. Tommasini

August 19, 2011

SPIE Optics \& Photonics San Diego, CA, United States August 21, 2011 through August 25, 2011 
This document was prepared as an account of work sponsored by an agency of the United States government. Neither the United States government nor Lawrence Livermore National Security, LLC, nor any of their employees makes any warranty, expressed or implied, or assumes any legal liability or responsibility for the accuracy, completeness, or usefulness of any information, apparatus, product, or process disclosed, or represents that its use would not infringe privately owned rights. Reference herein to any specific commercial product, process, or service by trade name, trademark, manufacturer, or otherwise does not necessarily constitute or imply its endorsement, recommendation, or favoring by the United States government or Lawrence Livermore National Security, LLC. The views and opinions of authors expressed herein do not necessarily state or reflect those of the United States government or Lawrence Livermore National Security, LLC, and shall not be used for advertising or product endorsement purposes. 


\title{
Advanced Gated X-Ray Imagers for Experiments at the National Ignition Facility
}

\author{
S. Glenn ${ }^{\text {a }}$, P. M. Bell ${ }^{\mathrm{a}}$, L. R. Benedetti ${ }^{\mathrm{a}}$, D. K. Bradley ${ }^{\mathrm{a}}$, J.Celeste ${ }^{\mathrm{a}}$, R.Heeter ${ }^{\mathrm{a}}$, C. Hagmann ${ }^{\mathrm{a}}$, \\ J.Holder ${ }^{a}$, N. Izumi ${ }^{a}$, J. D. Kilkenny ${ }^{b}$, J. Kimbrough ${ }^{a}$, G.A. Kyrala ${ }^{c}$, N. Simanovskaia ${ }^{a}$, R. \\ Tommasini $^{\mathrm{a}}$ \\ a Lawrence Livermore National Laboratory, 7000 East Avenue, Livermore, CA, USA 94550; \\ ${ }^{\mathrm{b}}$ General Atomics, 3550 General Atomics Court, San Diego USA 92121-1122; \\ 'Los Alamos National Laboratory, Los Alamos, NM USA 87545.
}

\begin{abstract}
$\mathrm{X}$-ray imaging is integral to the measurement of the properties of hot plasmas. To this end, a suite of gated x-ray imagers have been developed for use in a wide range of experiments at the National Ignition Facility (NIF). These instruments are sensitive to $\mathrm{x}$-rays over the range of $0.7-90 \mathrm{keV}$ and can acquire images at $20 \mathrm{ps}$ intervals for source intensities ranging over several orders of magnitude. We review the design, technology, and construction of these instruments and present recent results obtained from NIF experiments in which gated x-ray imagers have played a key role.

The radiation environment associated with Inertial Confinement Fusion (ICF) experiments presents unique challenges for x-ray imaging. We report on the performance of gated imagers that have been optimized for this harsh environment and describe diagnostics to be deployed in the near future that will provide x-ray images of imploding ICF capsules in the presence of backgrounds associated with neutron yields above $10^{16}$. Such images will provide crucial data that will enable even higher neutron yields and successful ignition.
\end{abstract}

Keywords: X-Ray Imaging, Framing Cameras, Lasers, NIF

\section{INTRODUCTION}

At the National Ignition Facility (NIF), inertial confinement fusion (ICF) experiments are underway in which capsules filled with cryogenic deuterium and tritium are heated and compressed to achieve nuclear fusion [1,2]. Gated x-ray imagers are extremely useful for such experiments since they provide important details about the evolution of the size, shape, and burn history of the compressed core [3]. Gated x-ray images reveal implosion asymmetries that would spoil ignition and help guide the optimal design of the laser drive, hohlraum, and capsule.

In indirect drive ICF experiments, a capsule containing $D T$ fuel is centered within a cylindrical cavity known as a hohlraum. As shown in figure 1, laser beams enter holes at either end of the hohlraum and heat the walls, resulting in xrays that rapidly heat ablator material near the surface of the capsule. The expanding ablator material produces a rocket effect that pushes the contents inward. As the capsule is compressed, hot plasma in the central region emits x-rays that are indicative of its size, shape, and temperature. At the NIF, these self-emission x-rays are typically imaged using $10 \mu \mathrm{m}$ pinholes $8-10 \mathrm{~cm}$ from the target. Framing cameras record pinhole images over adjacent lines of sight corresponding to $\sim 20 \mathrm{ps}$ intervals at magnifications of $12 \mathrm{X}$ to $15 \mathrm{X}$. Each pinhole image is analyzed for intensity, size, and shape, where the shape is usually expressed in terms of Legendre components of an intensity contour at a fixed fraction of the peak intensity.

The remaining sections review the gated imagers used at NIF to record time-resolved $\mathrm{x}$-ray images of implosions describe some of the challenges presented by increasing neutron yields. The final section discusses proposed a backlit imaging experiment that also requires sensitivity to $\mathrm{x}$-rays in the $60-200 \mathrm{keV}$ range. 


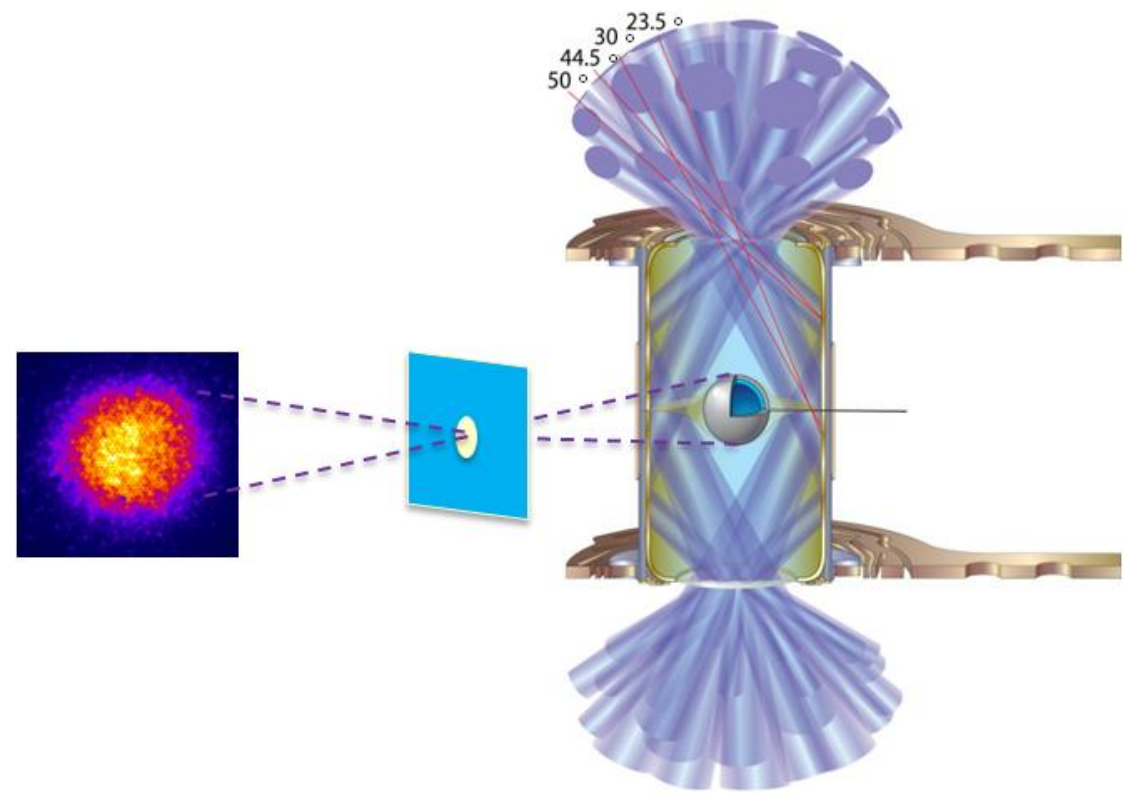

Figure 1. Cut-away view of a typical target used for NIF indirect-drive ICF experiments. Laser beams enter through holes at either end of the hohlraum, resulting in $\mathrm{x}$-rays that heat the capsule containing the DT fuel. Inset is a GXD image showing x-ray self-emission from the capsule core near the time of peak compression.

\section{GATED X-RAY IMAGERS ON THE NIF}

\subsection{Architecture}

The imaging architecture for $\mathrm{x}$-ray framing cameras used on the NIF is similar to that employed previously for ICF experiments at the NOVA and OMEGA facilities $[4,5]$. The gated microchannel plate (MCP) and proximity-focused phosphor imaging technology using in these instruments was developed and matured during the 1980's as it was incorporated into systems capable of reliable operation [6].

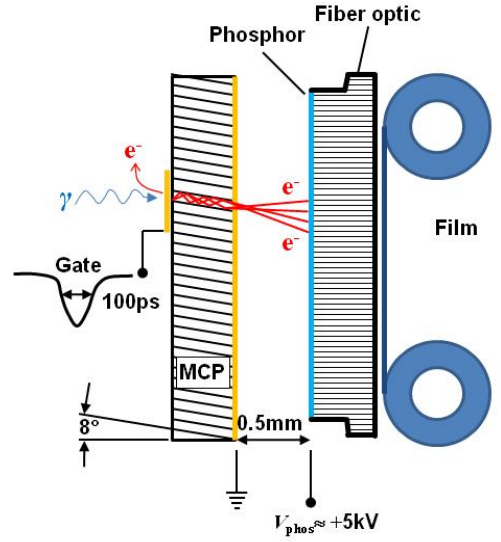

(a)

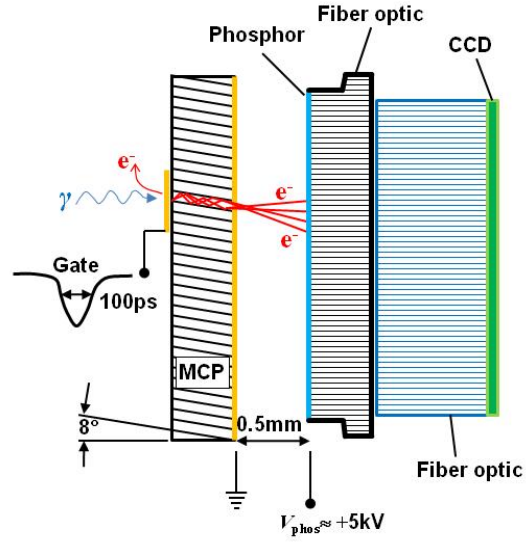

(b)

Figure 2. Gated imaging employing film (a) or CCD (b) to record images from phosphors. In both cases, a MCP is used to amplify secondary electrons ejected by x-rays incident on a gold photocathode strip. A voltage pulse on the strips provides time-dependent gain that gates the signal. Electrons from the MCP are proximity-focused on a phosphor screen, and the image is relayed to the recorder by a fiber optic block. 
As shown in figure 2, a gold photocathode strip coated on the MCP surface converts incident x-rays into electrons that are amplified within MCP pores when an accelerating voltage is applied. As the gate pulse travels along the strip, it effectively forms a "flying shutter" since signal is only produced in the region of the voltage pulse peak. Multiple strips can be deposited on the MCP, and usually consist of a $75 \AA$-thick adhesion layer under a $5000 \AA$ copper layer topped by a gold photocathode layer of thickness $1000 \AA$. In the four-strip configuration typically used at NIF, the strips have an active area $\sim 36 \mathrm{~mm}$ long and $7 \mathrm{~mm}$ wide. The channel pores generally have a length-diameter ratio $L / D \approx 40$ and are biased $8^{\circ}$ relative to normal to prevent x-rays from penetrating straight through the MCP. Gate voltages range from $700 \mathrm{~V}$ to $1000 \mathrm{~V}$ and are adjusted to provide the desired amount of gain. Since motion blurring can obscure important image features in ICF experiments, gate widths of 100ps or less are typically used, and gate widths as small as 40ps have been achieved with thin MCP's that allow electrons to transit the length of the pore before the end of the gate pulse [4].

ICF experiments typically use MCP's configured with two or four strips, with a separate pulser driving each strip at a configurable delay relative to the main trigger. Multiple pinholes produce identical images on MCP strips with small parallax, and the gate pulse timing is arranged to produce a time sequence of images.

Most x-ray framing cameras deployed at NIF undergo similar calibration procedures. A stable x-ray source is used for static measurements that characterize system resolution and gain as a function of MCP voltage. Historically, short-pulse UV lasers operating near 200nm have been used to characterize gate profiles as well as MCP gain and saturation [7-9]. Nanosecond-scale x-ray pulses from laser-produced plasmas are used to characterize the gain variation across strips.

Electrons produced by the MCP are accelerated across a gap of $0.3-0.5 \mathrm{~mm}$ with a potential of $\sim 5 \mathrm{kV}$ and are proximityfocused onto a phosphor screen to form an image which is relayed through a fiber-optic plate (FOP) to film or a CCDbased image recording system.

\subsection{The GXD Imager}

The Gated X-Ray Detector (GXD), developed at Los Alamos National Laboratory, is the primary gated imager used on NIF for experiments with neutron yields $\left(Y_{\mathrm{n}}\right)$ less than $10^{13}$. See reference [10] for a detailed description. Images are recorded electronically with a 16-megapixel CCD, and on-board computers perform monitoring and control functions. Except for the MCP, the entire instrument is operated at atmospheric pressure within an aluminum box mounted on the end of a Diagnostic Instrument Manipulator (DIM) within the NIF target chamber. The aluminum enclosure isolates sensitive components from the harsh NIF environment, allows convective cooling of electronics, and reduces the likelihood of high-voltage arcing due to partial vacuum. An imaging snout housing pinholes and filters is attached to the front of the GXD. After exiting the MCP, the gate pulses are delayed and multiplexed onto a single channel for crosstiming with the NIF laser.

The fiber optic plate forms the vacuum barrier between the GXD enclosure and the NIF target chamber. An important GXD feature is the use of a $100 \mu$ s voltage pulse to bias the phosphor to prevent high-voltage discharge due to gas originating from the interaction of the target and laser.

GXD's achieve $60 \mu \mathrm{m}$ resolution at the MCP image plane, corresponding to $5 \mu \mathrm{m}$ at the object when operated with $12 \mathrm{X}$ magnification. This resolution is reduced to $\sim 10 \mu \mathrm{m}$ by the pinhole imaging system. Typically, ICF experiments use filters that allow x-rays with energy above $\sim 7 \mathrm{keV}$ to reach the MCP, and $10 \mu \mathrm{m}$ pinholes in $75 \mu \mathrm{m}$-thick tantalum substrates are used to produce images. Tantalum collimators are used to reduce backgrounds due to hard x-rays that would otherwise penetrate the pinhole substrate.

\subsection{Effects of Neutrons on X-Ray Imaging}

ICF experiments at the NOVA and OMEGA laser demonstrated that careful consideration must be given to neutroninduced backgrounds associated with ICF experiments at NIF-class facilities [11-14]. Initial pre-ignition experiments with $T H D$-filled targets will have $\mathrm{Y}_{\mathrm{n}} \approx 10^{15}$, and a successful $D T$ ignition experiment with produce more than $10^{18}$ neutrons. Primary fusion neutrons and secondary particles adversely affect image quality through a variety of mechanisms that depend on the details of the detector and experimental configuration.

Fusion neutrons can produce charged particles $(p, \alpha)$, secondary neutrons, and gamma rays by both elastic and inelastic interactions with materials in the vicinity of the imager. The charged secondary particles create an optical background 
through scintillation in the phosphor, and gamma rays can further interact and create high-energy electrons that emit Cherenkov radiation in the FOP behind the phosphor $[15,16]$. In systems with CCD image recorders, neutrons, protons, and alpha particles, can interact with silicon in the sensitive region, resulting in recoil ions that create tracks and "stars" in the image. The effects of these localized background events can be mitigated through image-processing techniques [17]. A more diffuse background is created by energetic electrons that deposit energy in the sensitive region through ionization energy loss $[14,15]$. This background effectively raises the noise floor and is mainly responsible for limiting the useful range of CCD-based systems to experiments that result in neutron fluences less than $5 \times 10^{7} \mathrm{~cm}^{-2}$, corresponding to a $Y_{n}=10^{13}$ for a GXD fielded at the typical distance of $1.3 \mathrm{~m}$ from the target. CCD's also suffer cumulative performance degradation from exposure to high-yield shots that limits their useful lifetime.

At the NIF, a new radiation-hardened gated x-ray imagers are being used or introduced as yields are increased in ICF experiments [18]. Two such detectors, HGXI and ARIANE, are described in the following sections.

\subsection{HGXI}

The Hardened Gated X-ray Imager (HGXI) is designed for experiments with fusion neutrons yields up to $10^{15}$ [19]. Like the GXD, it is operated at atmospheric pressure within an aluminum "airbox" enclosure mounted on a DIM positioned such that the MCP is $1.3 \mathrm{~m}$ from the target. It differs from the GXD in several ways. The airbox contains no electronic components such as computers that are vulnerable to transient and cumulative radiation effects. Manual switches and relays are used to control the instrument, and monitoring is performed on analog signals routed away from the NIF target chamber. More importantly, the HGXI records images using TMAX 3200 film instead of a CCD, which enables operation at a higher $Y_{n}$ with acceptable image quality.

In contrast to CCD's, images recorded in photographic film do not exhibit localized features due to neutron-induced backgrounds. Rather, the main effects are increased noise and loss of sensitivity due to fogging, and the $Y_{n}$ limit is mainly due to optical backgrounds from the FOP and phosphor [15]. Backgrounds due to charged particle interactions in the film, Cherenkov light in the FOP, and phosphor scintillation are expected to contribute in the ratio 50:40:10, and film sensitivity to neutron-induced backgrounds has been measured to $30 \mathrm{X}$ lower than CCD's. An example is shown in figure 3, which contains a side-by-side comparison of approximately synchronous images acquired by a GXD and HGXI along different lines of sight for a shot with $Y_{n} \approx 2 \times 10^{13}$. Localized background features are clearly visible in the GXD (CCD) image, while the HGXI (film) image shows a smoothly varying background that has minimal influence on the apparent overall shape of the core emission. The GXD background is also higher due to its thicker FOP and additional fiber block used to transport the image to the CCD.

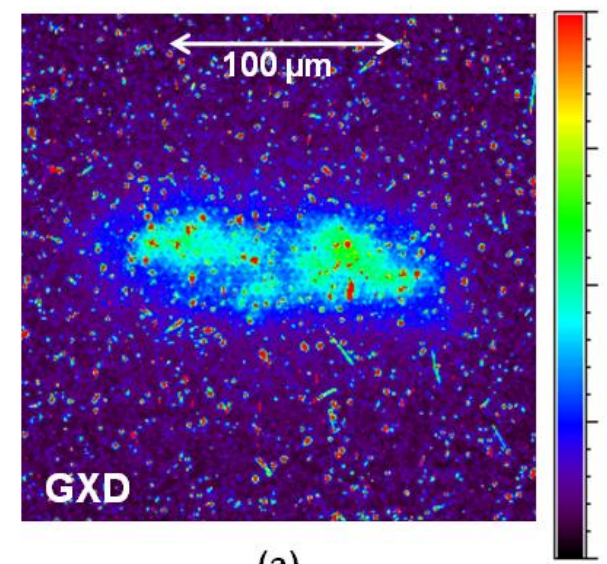

(a)

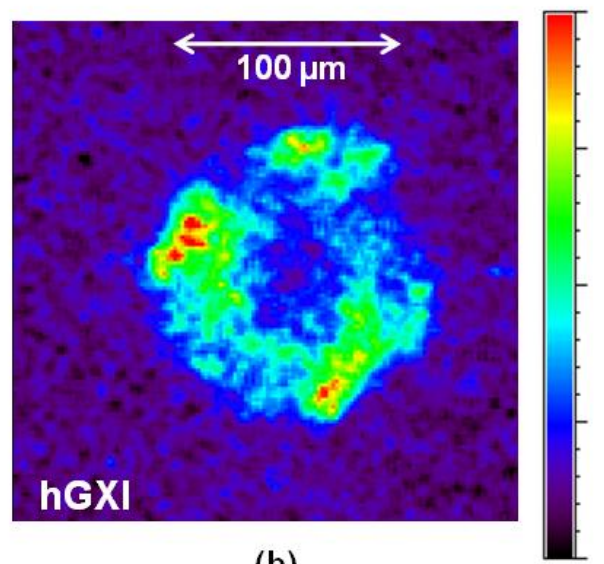

(b)

Figure 3. Comparison of (a) CCD-based GXD and (b) film-based hGXI images from NIF shot N110121-001-999 in which the capsule was filled with a THD mixture. The GXD line of sight was along the equator of the target, and the hGXI line of sight was along the axis of the hohlraum. In both cases, resolution is about $10 \mu \mathrm{m}$ at the image plane. 


\subsection{ARIANE}

For $Y_{n}>10^{15}$, further measures are required to acquire high-quality x-ray images of ICF capsules. As shown in figure 4, the ARIANE imager currently being commissioned is located outside of the NIF target chamber, $7 \mathrm{~m}$ from the target, and uses both film and electronic image recording techniques during progressive phases of deployment $[17,18]$. This arrangement confers several advantages: (1) the radiation field is lower as the distance to the target increases, (2) the increased distance results in a greater time difference between x-rays and neutrons at the detector, and (3) location outside the target chamber enables the use of shielding to reduce background levels. Similar to the HGXI, sensitive computerized control and monitoring electronics have been relocated far from the detector, or, in some cases, replaced by radiation-tolerant relay logic.

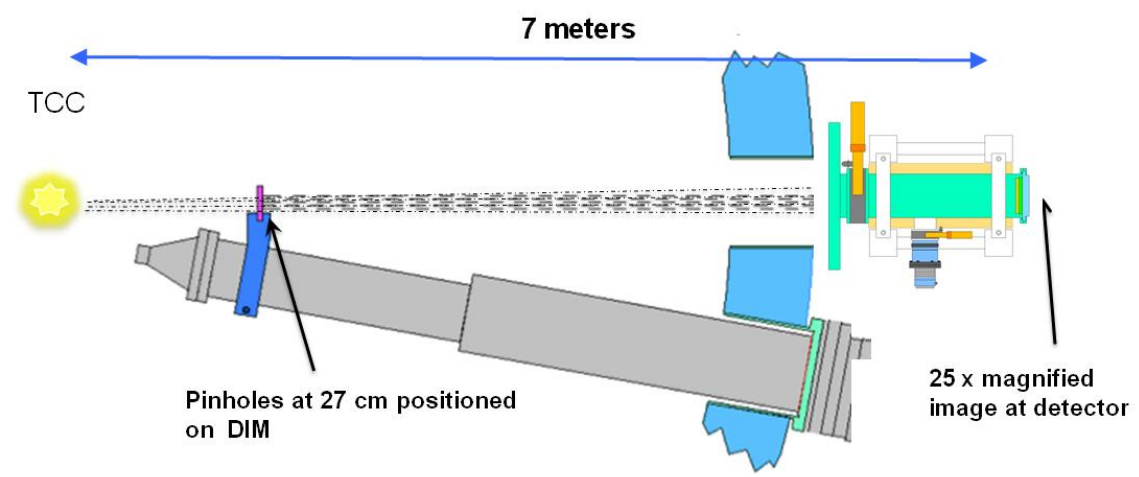

Figure 4. Schematic of the ARIANE detector with the MCP and phosphor components outside the target chamber at a distance of $7 \mathrm{~m}$ from target chamber center.

Early phases of ARIANE will use a CCD and, later, film to record images of the phosphor screen. As yields increase, a lens or fiber-optic system will relay the phosphor image to a shielded enclosure housing a CCD recording system. The shielding is expected to reduce the gamma and neutron backgrounds by factors of $100 \mathrm{X}$, and $1000 \mathrm{X}$, respectively [20]. Further background rejection will be achieved by exploiting the 140ns arrival difference between $\mathrm{x}$-rays and 14-MeV primary fusion neutrons. A gated optical intensifier will be placed in front of the CCD that will allow light from detected x-rays to be recorded, but will block background light originating in the detector or relay optics that is associated with the arrival of neutrons. This will require the use of phosphors such as P46 and P47 with decay times near 140ns. (The GXD and HGXI use P43 and P11 phosphors, respectively, with $\sim 1 \mathrm{~ms}$ decay times.) Recent measurements by Izumi [21] of phosphor decay times confirm that these phosphors are suitable for this method of background suppression.

\section{FUTURE EXTENSIONS AND APPLICATIONS}

As shown in Figure 5, the future ICF experiments at the NIF will involve imaging harder $(>60 \mathrm{kV}) \mathrm{x}$-rays and increasing yields. The following sections describe ARIANE extensions for operations at higher yield and a proposed Compton radiography experiment that uses hard x-rays to create areal density maps of the cold fuel surrounding the capsule core.

\subsection{Additional neutron-induced background suppression}

An advanced electronic readout system is being developed that promises to allow ARIANE operation at nearly full ignition yield $\left(Y_{n} \sim 10^{18}\right)$ [18]. In this scheme, a long-decay phosphor will be used to store the image for several hundred nanoseconds until the neutron-induced background subsides. Meanwhile, the readout sensor will be in a charge-dump state until being reset after the neutrons pass and subsequently recording the remaining light from the decaying phosphor. 
Additionally, increasing image signal-to-noise ratio through the use of higher phosphor voltages can mitigate neutroninduced backgrounds. MCP-phosphor detector assemblies are now being developed and tested that can support phosphor voltages up to $10 \mathrm{kV}$, which should more than double the light output of the phosphor relative to standard $5 \mathrm{kV}$ operation. This effort requires careful attention to material selection, surface quality, construction, and geometry to suppress discharging.

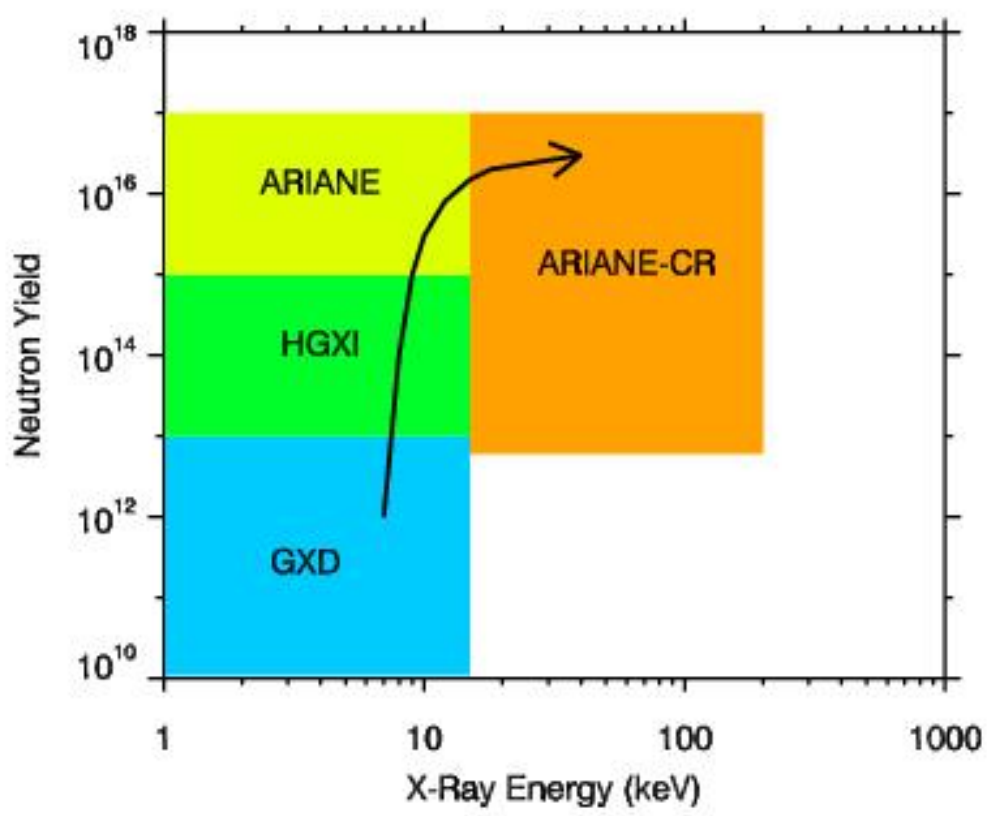

Figure 5. Anticipated trend for x-ray imaging in ICF experiments. Future experiments will image harder x-rays at increasing neutron yields.

Additionally, neutron-induced backgrounds can be mitigated by increasing image signal-to-noise ratio through the use of higher phosphor voltages. MCP-phosphor detector assemblies are now being developed and tested that can support phosphor voltages up to $10 \mathrm{kV}$, which should more than double the light output of the phosphor relative to standard $5 \mathrm{kV}$ operation. This effort requires careful attention to material selection and geometry to suppress discharging.

\subsection{Areal Density Measurements with Transmission Compton Radiography}

Point projection is an alternative to pinhole imaging that provides additional information that is not available from simply imaging self-emission from the core. Experiments at the OMEGA facility have demonstrated that it is possible to measure temporally- and spatially-resolved areal mass density of the capsule with better than $10 \%$ accuracy near peak compression [22]. The point projection technique uses short, high intensity laser pulses on $10 \mu \mathrm{m}$ gold wires to create point x-ray backlight sources that are typically separated in time by a few hundred picoseconds, and a gated x-ray imager is used to record the resulting radiograph of the target. In this case, backlighters provide temporal resolution, but a gated imager is required to reject early backgrounds due to x-rays resulting from laser-plasma interactions and late backgrounds from fusion neutrons. Imaging x-rays above $50 \mathrm{keV}$ reduces blurring due to refraction in the target and reduces background from self-emission through the use of high-pass filters. At these energies, the Compton process is the dominant $\mathrm{x}$-ray scattering mechanism for electron densities of interest, and the cross section is relatively constant in the range between $60 \mathrm{keV}$ and $200 \mathrm{keV}$. This has two benefits: the efficiency of the backlighter is enhanced since signal can be integrated over a wide energy range, and the experimental results are relatively insensitive to the shape of the backlighter spectrum. 
Figure 6 shows a possible implementation of implosion transmission radiography on NIF using twin ARIANE detectors to record signals from two backlight sources separated by 200ps along with simulated radiographs assuming $20 \mu \mathrm{m}$ spatial resolution, 10ps temporal resolution, and expected noise. The backlighters will be produced by the NIF Advanced Radiographic Capability (ARC), a dedicated subset of beams that will have capability of generating intensities of $10^{18} \mathrm{~W} / \mathrm{cm}^{2}$ [23]. A collimator will be used to isolate $\mathrm{x}$-rays from each of the two backlight sources, and an ARIANE detector with a single-strip MCP will be located along each of the two lines of sight. Radiographs will be processed to correct any instrumental effects and the fuel areal density for cylindrically symmetric implosions will be inferred using established techniques, e.g., Abel inversion.

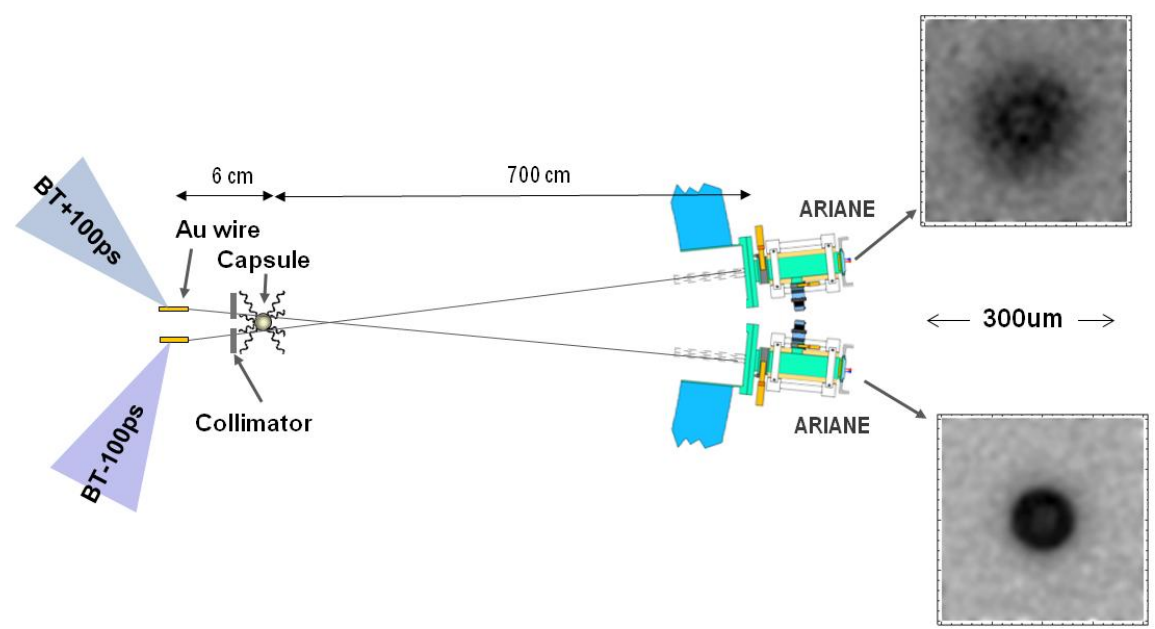

Figure 6. Experimental setup for Compton radiography measurements at NIF using twin ARIANE detectors. Also shown are simulated radiographs with $20 \mu \mathrm{m}$ spatial resolution and $10 \mathrm{ps}$ temporal resolution.

\section{CONCLUSION}

Gated $x$-ray imagers have provided important time-resolved information about the shape and size of implosions in ICF experiments at the NIF and will continue to do so as neutron yields increase. For experiments with low yield $\left(Y_{n}<10^{13}\right)$, the GXD is used with electronic readout and automated controls and monitoring. At intermediate yields $\left(10^{13}<Y_{n}<10^{15}\right)$, experiments employ the film-based hGXI, and higher yield experiments will use the ARIANE detector. Extensions to the ARIANE platform will allow it to acquire images at near-ignition yields, and imaging x-rays at energies above 50 $\mathrm{keV}$ will enable point-projection Compton radiography experiments that measure fuel density. All of these capabilities will contribute to the optimization of conditions for ICF and lead to successful ignition.

\section{ACKNOWLEDGEMENTS}

This work was performed under the auspices of the U.S. Department of Energy by Lawrence Livermore National Laboratory under Contract DE-AC52-07NA27344. 


\section{REFERENCES}

[1] J.D. Lindl, "Development of the indirect-drive approach to inertial confinement fusion and the target physics basis for ignition and gain", Physics of Plasmas 2, 3933 (1995).

[2] Miller, G. H, Moses, E. I., and Wuest, C. R., "The National Ignition Facility: enabling fusion ignition for the 21st century", Nuclear Fusion 44, S228 (2004).

[3] Kyrala G. A., Dixit, S., Glenzer, S., Kalantar, D., et al, "Measuring symmetry of implosions in cryogenic Hohlraums at the NIF using gated x-ray detectors", Review of Scientific Instruments 81, 10E316 (2010).

[4] Bradley, D.K., Bell, P.M., Landen, O.L., Kilkenny, J.D., Oertel, J., "Development and characterization of a pair of 30-40 ps x-ray framing cameras", Review of Scientific Instruments 66, 716 (1995).

[5] Budil, K.S., Perry, T.S., Bell, P.M., Hares J.D., Miller, P.L., Peyser, T.A., Wallace, R., Louis, H., and Smith, D.E., "The flexible x-ray imager", Review of Scientific Instruments 67, 485 (1996).

[6] Kilkenny, J. D., "High speed proximity focused X-ray cameras", Laser and Particle Beams 9, 49 (1991).

[7] Landen, O. Abare, A., Hammel, B. A., Bell, P. M., Bradley, D. K., "Detailed measurements and shaping of gate profiles for microchannel-plate-based x-ray framing cameras", Proceedings of the SPIE Vol. 2273, 253 (1994).

[8] Kruschwitz, C.A., Wu, M., Moy, K., Rochau, G., "Measurement and modeling of pulsed microchannel plate operation", Review of Scientific Instruments 79, 10E911 (2008).

[9] Landen, O., Oertel, J.A., Bell, P. M., Satariano, J.J., Bradley, D. K., "Gain uniformity, linearity, saturation, and depletion in gated microchannel-plate x-ray framing cameras", Proceedings of the SPIE Vol. 2002, 2 (1993)

[10] Oertel, J., et al., "Gated x-ray detector for the National Ignition Facility", Review of Scientific Instruments 77, 10E308 (2006).

[11] Lerche, R. A., "Neutron-induced noise in National Ignition Facility-class diagnostic instruments", Review of Scientific Instruments 68, 631 (1997).

[12] Jaanimagi, P. A., Boni, R., Keck, R. L., "Neutron-induced background in charge-coupled device detectors”, Review of Scientific Instruments 72, 801 (2001).

[13] Bourgade, J. L., et al, "New constraints for plasma diagnostics development due to the harsh environment of MJ class lasers", Review of Scientific Instruments 75, 4204 (2004).

[14]Bourgade, J. L., "Diagnostics hardening for harsh environment in Laser Mégajoule", Review of Scientific Instruments 79, 10F301 (2008).

[15] Izumi, N., et al., "Experimental study of neutron induced background noise on gated x-ray framing cameras", Review of Scientific Instruments 81, 10E515 (2010).

[16] Hagmann, C., et al., "Radiation Induced Noise in X-Ray Imagers for High-Yield Inertial Confinement Fusion Experiments", these proceedings.

[17] Smalyuk, V., et al., "X-Ray Imaging in an Environment with High-Neutron Background on the National Ignition Facility", these proceedings.

[18] Bell, P.M., al., "Radiation hardening of gated x-ray imagers for the National Ignition Facility", Review of Scientific Instruments 81, 10E540 (2010).

[19] Glenn, S., al., "A hardened gated x-ray imaging diagnostic for inertial confinement fusion experiments at the National Ignition Facility", Review of Scientific Instruments 81, 10E539 (2010).

[20] Hagmann, C., et al., "Modeling of neutron induced backgrounds in x-ray framing cameras", Review of Scientific Instruments 81, 10E514 (2010).

[21] N. Izumi, et al., "Efficiency and decay time measurement of phosphors for x-ray framing cameras usable in harsh radiation background", these proceedings.

[22] Tommasini, R., et al., "Development of Compton radiography of inertial confinement fusion implosions", Physics of Plasmas 18, 056309 (2011)

[23] Crane, J. K, et al., "Progress on Converting a NIF Quad to Eight, Petawatt Beams for Advanced Radiography", Journal of Physics: Conference Series 244, 032003 (2010) 